\title{
CLINICAL RESEARCH ARTICLE Origins of neonatal leptin deficiency in preterm infants
}

\author{
Baiba Steinbrekera ${ }^{1}$, Tarah T. Colaizy ${ }^{1}$, Lauren K. Vasilakos ${ }^{1}$, Karen J. Johnson ${ }^{1}$, Donna A. Santillan ${ }^{2}$, Sarah E. Haskell ${ }^{1}$ and \\ Robert D. Roghair ${ }^{1}$
}

BACKGROUND: Cord blood leptin increases with advancing gestation. Preterm delivery leads to premature separation from the maternal and placental leptin source predisposing infants to postnatal leptin deficiency, but this has not been fully described. METHOD: Blood leptin levels were measured for infants born before 33 weeks gestation daily for the first 2 days, then weekly until 36 weeks postmenstrual age (PMA). Cord blood was obtained to provide gestational age (GA)-specific standards.

RESULTS: Cord blood leptin levels were positively associated with GA at birth, maternal body mass index (BMI) and pregnancy weight gain (all $P<0.05$ ). Following birth, infant leptin levels decreased rapidly (74\% decrease within $48 \mathrm{~h}$ ). The extent of this decline correlated with GA $(P<0.05)$. Postnatal leptin began to increase by 33-36 weeks PMA, but remained below cord blood leptin levels $(P<0.01)$. At 36 weeks PMA, leptin levels were influenced by infant's weight and sex $(P<0.01)$, with females having higher leptin levels $(1213 \mathrm{pg} / \mathrm{ml}$ vs. $984, P<0.05)$.

CONCLUSION: Cord blood leptin is influenced by maternal weight gain and BMI, suggesting an important role for trans-placental leptin delivery. Preterm delivery leads to sustained leptin deficiency through 36 weeks PMA, with the most premature male infants facing the longest and harshest deficiency.

Pediatric Research (2019) 85:1016-1023; https://doi.org/10.1038/s41390-019-0359-y

\section{INTRODUCTION}

The second half of pregnancy plays a key role in ongoing cell differentiation and organ maturation. Hormones derived from the maternal circulation and placenta indirectly and directly contribute to this critical phase of organogenesis. Alterations in the supply of hormones and other trophic signals to the infant can thus disrupt fetal development. For example, as an indirect consequence of altered maternal metabolism, cardiomyopathy and impaired brain development have been reported in infants of diabetic mothers. ${ }^{1}$ Because of their premature separation from maternal and placental influences, preterm infants are at an increased risk of detrimental long-term outcomes.

It is widely known that preterm birth is a leading risk factor for adult metabolic syndrome and cognitive deficit. The rapid development that typically occurs during the third trimester is slowed by preterm delivery, and a crucial window of opportunity can pass while the infant is in a battle for extrauterine survival. These concerns have prompted investigations to uncover critical factors, including neurotrophic hormones, that could promote neuromaturation in the absence of direct maternal and placental influences. Our basic science investigations have confirmed the seminal observations of others in demonstrating an important neurodevelopmental role of leptin. ${ }^{2}$

Leptin is a pleiotropic hormone involved in adult metabolic regulation, as it affects both feeding behavior and energy expenditure. ${ }^{3}$ Less well known, but perhaps more important, is leptin's role as a neurotrophic hormone during development. ${ }^{4,5}$ Cord blood studies demonstrate that fetal leptin levels increase with advancing gestation ${ }^{6,7}$ and are dependent on both transplacental delivery and placental leptin production until around 32 weeks gestation. ${ }^{8}$ Only after $30-32$ weeks, when the fetus starts to accrue significant amounts of adipose tissue, does the fetus become the main contributor to its own circulating leptin levels. ${ }^{8}$

Unfortunately, delivery before 32 weeks can deprive the infant of maternal and placental leptin supply before endogenous production increases. This can lead to leptin deficiency during a time of rapid growth and development. Late preterm and term male infants have lower leptin levels than female infants, ${ }^{9-11}$ predisposing them to adverse developmental outcomes. Limited investigations have shown leptin levels may be higher in breastfed versus formula-fed term infants. ${ }^{12}$ We designed this study to fill several knowledge gaps by describing preterm infant leptin levels from periviable to near term gestation with consideration for factors that might affect leptin levels in this population. We hypothesized that premature birth rapidly results in leptin deficiency with the greatest effect in male infants born at earlier gestations, and this deficiency may be attenuated in infants with greater amounts of breast milk intake.

\section{MATERIALS AND METHODS}

All infants without congenital anomalies born between $220 / 7$ and $326 / 7$ weeks gestation admitted to the University of lowa Stead Family Children's Hospital Neonatal Intensive Care Unit (NICU) between January 2016 and August 2017 were eligible for enrollment. Gestational age (GA) was assessed by last menstrual period or early ultrasound. Only infants born before 33 weeks gestation were eligible, since the primary aim of the study was to evaluate leptin changes in infants who have not yet accreted significant adipose tissue. Infants were prospectively enrolled in three predefined cohorts, (1) those born at 22 to 25 weeks GA, defined as "periviable" gestations, (2) those born at 26 to 29 weeks

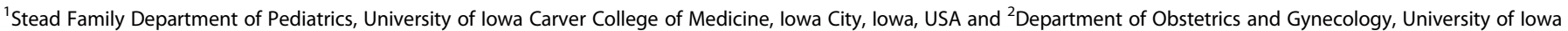
Carver College of Medicine, lowa City, lowa, USA

Correspondence: Robert D. Roghair (robert-roghair@uiowa.edu)
}

Received: 16 November 2018 Revised: 16 February 2019 Accepted: 26 February 2019

Published online: 7 March 2019 


\begin{tabular}{|c|c|c|c|c|c|}
\hline & All infants $N=142$ & $22-25$ weeks $\mathrm{N}=29$ & $26-29$ weeks $N=51$ & $30-32$ weeks $N=62$ & $P$ \\
\hline Birth weight (g) & $1186(883,1610)$ & $624(554,757)$ & $1075(966,1223)$ & $1655(1370,1914)$ & $<0.01$ \\
\hline Birth weight z-score & $0.14(-0.47,0.53)$ & $0.13(-0.66,0.49)$ & $0.13(-0.15,0.59)$ & $0.18(-0.63,0.53)$ & 0.32 \\
\hline $10^{\text {th }}-90^{\text {th }}$ & $124 / 142$ & $25 / 29$ & $45 / 51$ & $54 / 62$ & \multirow{2}{*}{0.36} \\
\hline$>90^{\text {th }}$ & $9 / 142$ & $2 / 29$ & $5 / 51$ & $2 / 62$ & \\
\hline Birth head circumference $(\mathrm{cm})$ & $27(24,29)$ & $21(20,23)$ & $26(25,27)$ & $30(28,31)$ & $<0.01$ \\
\hline Male sex & $75 / 142$ & $17 / 29$ & $22 / 51$ & $36 / 62$ & 0.30 \\
\hline Antenatal steroids, any & $136 / 142$ & $28 / 29$ & $47 / 51$ & $61 / 62$ & 0.25 \\
\hline 4 & $21 / 136$ & 0 & $8 / 47$ & $13 / 61$ & $<0.01$ \\
\hline \multicolumn{6}{|l|}{ Time between antenatal steroids and birth (hours) } \\
\hline$\leq 24$ & $41 / 136$ & $11 / 28$ & $12 / 47$ & $18 / 61$ & \multirow[t]{3}{*}{0.90} \\
\hline $25-48$ & $10 / 136$ & $1 / 28$ & $4 / 47$ & $5 / 61$ & \\
\hline$>48$ & $85 / 136$ & $16 / 28$ & $31 / 47$ & $38 / 61$ & \\
\hline Maternal age (years) & $29(25,33)$ & $27(24,35)$ & $28(25,31)$ & $30(27,33)$ & 0.20 \\
\hline $1^{\text {st }}$ trimester maternal body mass index (BMI) & $26.2(21.7,32.8)$ & $27.8(23.4,35.0)$ & $25.0(21.0,32.7)$ & $26.2(21.9,32.7)$ & 0.24 \\
\hline Maternal BMI at delivery & $30.1(27.2,38.0)$ & $30.0(26.8,36.6)$ & $29.4(27.3,38.2)$ & $31.1(26.7,38.0)$ & 0.93 \\
\hline Maternal BMI change & $3.3(2.0,4.9)$ & $2.6(1.2,3.4)$ & $3.0(1.3,6.0)$ & $3.8(2.6,4.9)$ & $<0.05$ \\
\hline Maternal diabetes, any & $15 / 141$ & $2 / 29$ & $5 / 50$ & $8 / 62$ & 0.67 \\
\hline Stage 1 & $22 / 31$ & $10 / 17$ & $10 / 12$ & $2 / 2$ & \multirow[t]{3}{*}{0.62} \\
\hline Stage 2 & $8 / 31$ & $6 / 17$ & $2 / 12$ & $0 / 2$ & \\
\hline Stage 3 & $1 / 31$ & $1 / 17$ & $0 / 12$ & $0 / 2$ & \\
\hline Laser therapy for ROP & $1 / 30$ & $1 / 17$ & $0 / 11$ & $0 / 2$ & 1.0 \\
\hline
\end{tabular}

Continuous variables, expressed as medians (interquartile range), were analyzed by Kruskal-Wallis Test while categorical variables, expressed as proportions, were analyzed by Fisher's Exact or Chi-Square Tests

GA, and (3) those born at 30 to 32 weeks GA. Ethical approval was obtained from the University of lowa Institutional Review Board (IRB \#201510835). Informed parental consent was obtained prior to enrollment in the study. Cord blood samples were obtained through the University of lowa Maternal Fetal Tissue Bank (IRB \#200910784). ${ }^{13}$

Cord blood was obtained for inborn infants. An additional 14 cord blood samples were obtained from infants born at $330 / 7$ to 36 6/7 weeks gestation to compare infant postnatal leptin levels with cord blood levels at 33 to 36 weeks PMA. The first infant blood sample was obtained between 6 to $24 \mathrm{~h}$ after birth, followed by daily sampling for the first 2 days of life and then weekly sampling until 36 weeks PMA. All blood was obtained during previously scheduled lab draws. After the first week of life, a vast majority of samples were obtained with morning labs at 0400 , one hour after the start of an enteral feeding.
A total of 200 microliters of blood was collected in EDTA tubes and processed within $4 \mathrm{~h}$. Plasma was stored at $-80^{\circ} \mathrm{C}$. Samples were analyzed using a customized magnetic bead assay (Millipore Sigma, Burlington, MA, USA) on BioPlex 200 with BioPlex manager 6.1 software (Bio-Rad, Hercules, CA, USA). All samples were run in duplicate with our intra-assay correlation coefficient $>0.9$ and intra-assay coefficient of variation of $7.4 \%$. If the leptin level was below the limit of detection $(41.0 \mathrm{pg} / \mathrm{ml})$, a value equal to the limit of detection divided by the square root of two (i.e., $29.0 \mathrm{pg} / \mathrm{ml}$ ) was assigned. Each assay included seven standards and a quality control to ensure lot-to-lot consistency.

At clinician's discretion, all infants born before 30 weeks gestation were begun on parenteral nutrition, 10\% Premasol (Baxter, Deerfield, IL, USA) within $24 \mathrm{~h}$ of birth and $20 \%$ Intralipid (Fresenius Cabi AB, Uppsala, Sweden) was added on day one. Infants born between 30 and 32 weeks gestation were initially 
placed on intravenous dextrose to prevent hypoglycemia and parenteral nutrition was started once central access was obtained. Enteral feeds were started on day one at $10-20 \mathrm{ml} / \mathrm{kg} /$ day and advanced by $10-20 \mathrm{ml} / \mathrm{kg} /$ day as tolerated to a goal of $140-150$ $\mathrm{ml} / \mathrm{kg} /$ day. Enfamil Human Milk Fortifier (Mead Johnson Nutrition, Chicago, IL, USA) was added to breast milk with goal caloric intake of $110-120 \mathrm{kcal} / \mathrm{kg} / \mathrm{day}$. According to parental wishes, infants were fed mother's milk, donor milk, or preterm commercial formula.

Maternal and infant demographic and clinical data were retrospectively collected using electronic medical records (Epic, Verona, WI, USA). Weekly data based on the last seven day period prior to sample acquisition were collected for each infant. Data included weight, glucose levels, dexamethasone exposure, head circumference, total calories received, and form of nutrition. We also collected data on bronchopulmonary dysplasia (BPD), defined as oxygen use at 36 weeks PMA to maintain oxygen saturations above $90 \%,{ }^{14}$ and retinopathy of prematurity (ROP), based on the worst ROP stage observed by ophthalmologist prior to maturation of retinas. All data were recorded and stored in REDCap version 8.3.2 (Vanderbilt, TN, USA).

Statistical analysis was performed using SAS version 9.4 (SAS Institute Inc., Cary, NC, USA) and GraphPad Prism version 7.0 (GraphPad Software, La Jolla, CA, USA). The clinical characteristics of the study population and leptin concentrations are expressed as median (interquartile range-IQR) or proportion. Infants were divided into three GA groups and characteristics were compared using Fisher's Exact or Chi-Square tests for categorical variables, and Kruskal-Wallis Test for continuous variables. Significance between different time points across different GA groups was tested with Two-Way ANOVA with Holm-Sidak method for multiple comparisons. Simple linear regression and generalized linear modeling were used to determine the relationship of leptin levels in cord blood and at 36 weeks GA with predictor variables. Univariate regression was performed for each predictor, and then the results were used to build multivariate models for leptin levels at those two time points. Statistical significance was defined by $P<0.05$.

\section{RESULTS}

A total of 142 infants with a median GA of 29.4 weeks were prospectively enrolled. There were 29 infants born at $220 / 7$ to 25 6/7 weeks, 51 infants born at $260 / 7$ to $296 / 7$ weeks, and 62 infants born at $300 / 7$ to $326 / 7$ weeks. Infant and maternal baseline characteristics are provided in Table 1. As expected, birth weight and head circumference differed based on GA at birth, but median Z-score for weight was similar across all GA groups $(0.13$, 0.13 , and 0.18 , respectively, $P=0.32$ ), with most infants $(87 \%)$ appropriate for GA (AGA). Maternal first trimester BMI and BMI prior to delivery were similar across GA groups, but as anticipated, $\mathrm{BMI}$ increase during pregnancy was higher in mothers who delivered later in pregnancy. Most mothers (96\%) received antenatal steroids, with $63 \%$ of those receiving a full course (two doses given $48 \mathrm{~h}$ prior to delivery). There were no differences between the groups for maternal and labor complications, except maternal preeclampsia predominated in the older GA infant groups, and the highest cesarean section rate $(86 \%)$ was seen in the 26-29 week cohort. Infant comorbidities, including BPD and ROP predominated in the younger GA infant groups, with the highest rates seen in infants born at 22-25 weeks gestation. The median time to reach goal caloric intake by enteral feeds alone varied with GA at birth with the 22-25 week cohort arriving at full feeds at a median postnatal age of 52 days, the 26-29 week cohort at 35 days, and the 30-32 week cohort at 26 days. The median PMA to achieve full feeds was 32,33 , and 35 weeks, respectively.

A total of 80 cord blood samples were collected from our study population. Due to insufficient sample quantity or hemolysis, 11 samples were not able to be analyzed. Two cord blood samples (one of $90,458 \mathrm{pg} / \mathrm{ml}$ within the 26-29 week group and one of $17,459 \mathrm{pg} / \mathrm{ml}$ within the 30-32 week group) were excluded as outliers. Based on the remaining 67 samples, median cord blood leptin was $1303 \mathrm{pg} / \mathrm{ml}$. Cord blood leptin predictor variables and their median or proportion are reported in Table 2. Univariate analysis revealed that GA at birth, birth weight, birth head circumference, maternal BMI at time of delivery, and maternal BMI change during pregnancy were all independent predictors of cord blood leptin (Table 2). Cord blood leptin did not differ for infants born by cesarean section versus vaginal delivery $(1186 \mathrm{pg} / \mathrm{ml}$ versus $1165 \mathrm{pg} / \mathrm{ml}, P=0.31$ ). While exposure to a complete course of antenatal steroids was associated with higher cord blood leptin levels than seen after an incomplete course of antenatal steroids, when adjusted for $\mathrm{GA}$, this did not approach statistical significance ( $1466 \mathrm{pg} / \mathrm{ml}$ versus $666 \mathrm{pg} / \mathrm{ml}, P=0.30$ by 2 way ANOVA). Multivariate regression analysis was performed with those significant variables plus infant's sex and maternal preeclampsia as potential covariates. Infant's birth weight and head circumference were excluded from multivariate analysis as they strongly

\begin{tabular}{|c|c|c|c|c|c|}
\hline & Median (IQR) or proportion & Univariate beta & Univariate $P$ & Multivariate beta & Multivariate $P$ \\
\hline Birth weight (g) & $1265(955,1728)$ & 0.7 & $<0.05$ & & \\
\hline Birth weight z-score & $0.08(-0.39,0.62)$ & -92.0 & 0.67 & & \\
\hline $1^{\text {st }}$ trimester maternal body mass index (BMI) & $26.3(22.7,32.9)$ & 26.7 & 0.23 & & \\
\hline Maternal BMI at delivery & $30.9(27.4,36.7)$ & 46.8 & $<0.05$ & 47.1 & $<0.05$ \\
\hline Maternal BMI change & $3.8(2.5,4.9)$ & 198.9 & $<0.01$ & 176.8 & $<0.05$ \\
\hline Maternal diabetes, any & $10 / 66$ & 92.4 & 0.55 & & \\
\hline Maternal hypertension & $8 / 67$ & 93.4 & 0.61 & & \\
\hline
\end{tabular}

The multivariate regression analysis included the significant variables from univariate analysis plus sex and maternal preeclampsia as potential covariates. Given their strong correlation with gestational age at birth, birth weight and head circumference were excluded from the multivariate analysis 


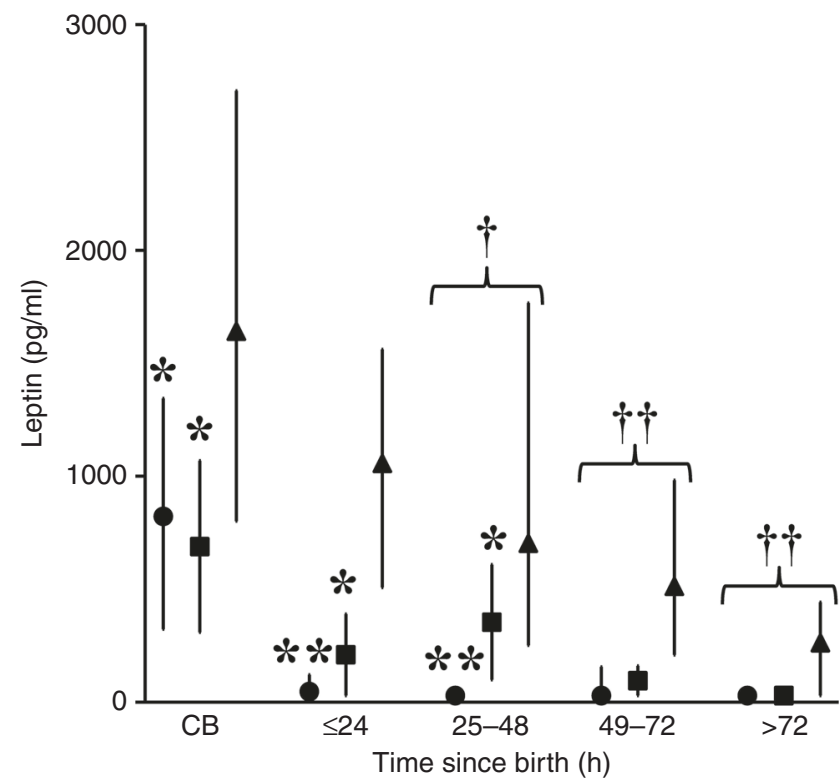

Fig. 1 Plasma leptin was measured in cord blood and postnatal blood obtained within a week of preterm delivery. Samples were obtained from infants born at a completed gestational age of 22-25 weeks (circles, $N=29$ ), 26-29 weeks (squares, $N=51$ ), or 30-32 weeks (triangles, $N=62$ ). Following a cord blood sample (CB), the first postnatal sample was obtained 6 to $24 \mathrm{~h}$ after birth, followed by daily sampling for the first 2 days and a final early sample obtained 3 to 7 days after delivery. Compared to infants born at 30-32 weeks, those born at earlier gestations had lower leptin concentrations in both cord blood and postnatal blood obtained within 2 days of delivery $\left({ }^{*} P<0.05\right.$ or $\left.{ }^{*} P<0.01\right)$. Compared to cord blood levels, leptin concentrations were significantly decreased within $25-48 \mathrm{~h}$ of delivery and this leptin deficit persisted throughout the first week of life $(+P<0.05$ or $+\dagger P<-0.01)$. Data are presented as median (IQR) and analysis utilized two-way ANOVA with Holm-Sidak method for multiple comparisons

correlate with GA at birth. Based on generalized linear modeling, GA at birth, maternal BMI at delivery, and maternal pregnancy BMI change continued to predict cord blood leptin levels (Table 2).

Eleven percent of the anticipated longitudinal infant samples were lost either due to insufficient sample quantity or missed sample collection, due to no scheduled lab draws that week. After delivery, leptin levels decreased within $24 \mathrm{~h}$ of birth (Fig. 1) and, compared to cord blood levels, this decrease became statistically significant at $25-48 \mathrm{~h}$. Leptin level drop (the difference between cord blood and postnatal values) correlated with GA and time since birth, with an exaggerated $96 \%$ decrease from cord blood levels seen in infants born at 22-25 weeks by $25-48 \mathrm{~h}$ of life $(823$ to $29 \mathrm{pg} / \mathrm{ml}, P<0.01)$. Infants born at 22-25 weeks or 26-29 weeks gestation had significantly lower leptin $(46 \mathrm{pg} / \mathrm{ml}$ and $210 \mathrm{pg} / \mathrm{ml}$, respectively) than infants born at $30-32$ weeks gestation ( $1057 \mathrm{pg} / \mathrm{ml}$ ) within $24 \mathrm{~h}$. Only after $48 \mathrm{~h}$ of life, leptin levels were similar across all gestations for the rest of the first week of life.

Postnatal leptin levels after the first week of life are represented in Fig. 2. Following the initial leptin level decrease, the 22-25 week and 26-29 week cohorts exhibited minimal leptin level rise until 33-36 weeks PMA. These two younger GA infant groups exhibited a more dramatic leptin increase between 30-32 and 33-36 weeks PMA when compared to that seen in 30-32 week group (22-25 week group change: 120 to $883 \mathrm{pg} / \mathrm{ml}, 26-29$ week group change: 177 to $671 \mathrm{pg} / \mathrm{ml}, 30-32$ week group change: 325 to $570 \mathrm{pg} / \mathrm{ml} ; P<0.01)$. Despite leptin level rise seen in all GA groups, postnatal leptin levels remained far below

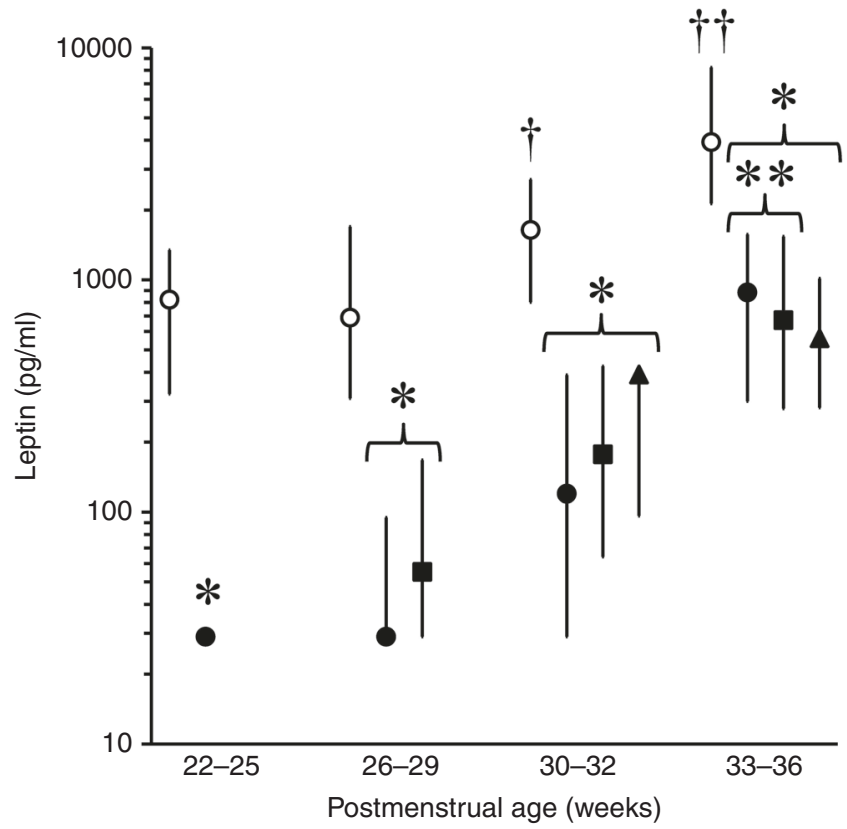

Fig. 2 Longitudinal plasma leptin concentrations were measured beginning one week after delivery and culminating at a postmenstrual age (PMA) of 36 weeks with comparison to prospectively obtained cord blood concentrations. Samples were obtained from infants born at a completed gestational age of 22-25 weeks (solid circles, $N=29$ ), $26-29$ weeks (squares, $N=51$ ), or 30-32 weeks (triangles, $N=62$ ). Cord blood samples (open circles) were obtained from infants born at $22-25$ weeks $(N=15), 26-29$ weeks $(N=19)$, 30-32 weeks $(N=33)$, or 33-36 weeks $(N=14)$. Cord blood leptin levels increased with advancing gestational age $(+P<0.05$ versus cord blood obtained at $22-25$ weeks, $+\uparrow P<-0.01$ versus cord blood obtained at 22-25, 26-29, and 30-32 weeks). Compared to cord blood levels, postnatal leptin concentrations remained significantly low through 36 weeks PMA $(* P<0.01$ versus cord blood at the corresponding PMA). By 33-36 weeks PMA, infants born at 22-25 or 26-29 weeks achieved higher leptin levels than those born at $30-32$ weeks $(* * P<0.01)$. Samples with a leptin level below the limit of detection $(41 \mathrm{pg} / \mathrm{ml})$ were assigned a value equal to the limit of detection divided by the square root of two $(29 \mathrm{pg} / \mathrm{ml})$. Data are presented on a logarithmic scale as median (IQR) and analysis utilized two-way ANOVA with Holm-Sidak method for multiple comparisons

cord blood leptin levels at all time points through 33-36 weeks PMA $(P<0.01)$.

Because longitudinal leptin levels may be influenced by insulin, corticosteroids, and gender, additional data were extracted from the electronic medical record. Average daily glucose levels, obtained at the same time as the research samples, varied from 75 to $115 \mathrm{mg} / \mathrm{dl}$ with an overall mean of 96 and a standard deviation of 25 . Only $3.6 \%$ of the glucose levels were above 150 $\mathrm{mg} / \mathrm{dl}$ and only $0.8 \%$ of values were less than $50 \mathrm{mg} / \mathrm{dl}$. None of the infants received insulin therapy. Overall, 10 of 29 infants in the 22-25 week cohort and 5 of 51 infants in the 26-29 week cohort received postnatal dexamethasone for ventilator-dependent lung disease. Among those 15 infants, leptin levels during dexamethasone administration were not significantly different from leptin levels obtained in the weeks before or after dexamethasone administration $(387 \mathrm{pg} / \mathrm{ml}$ versus $292 \mathrm{pg} / \mathrm{ml}, P=0.16$ by paired $T$ test). Figure 3 provides longitudinal leptin levels for female versus male infants. Independent of GA at delivery, from a PMA of 26 to 36 weeks, female infants had significantly higher leptin levels than male infants.

One hundred eighteen infants completed the study period reaching 36 weeks PMA. Seven infants were lost due to death and 


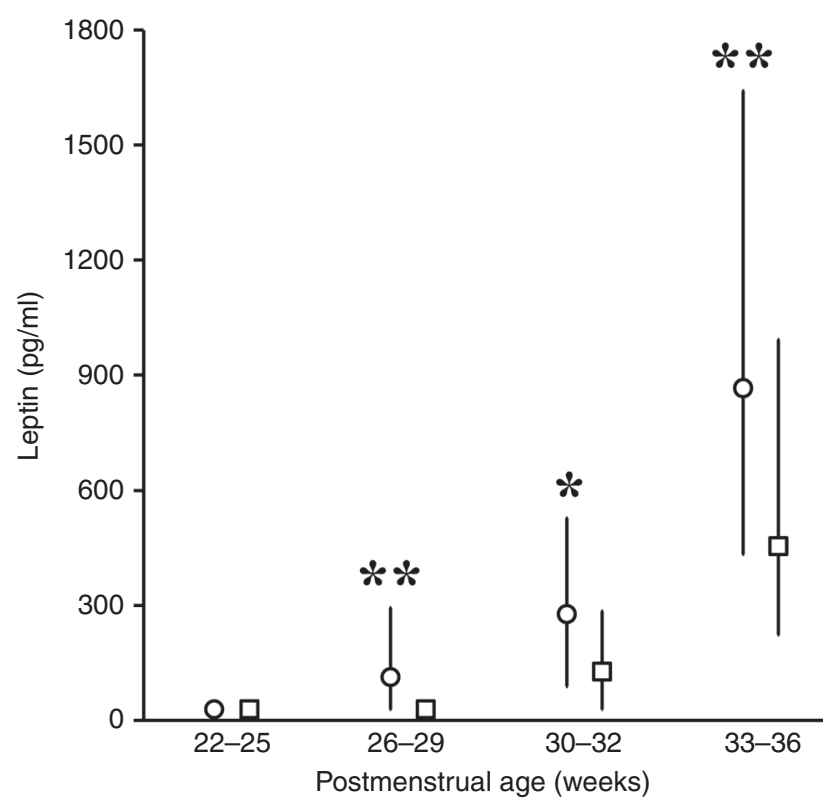

Fig. 3 Longitudinal plasma leptin concentrations were measured for female and male infants beginning one week after delivery and culminating at a postmenstrual age (PMA) of 36 weeks. ANOVA was utilized to compare samples obtained from 77 female infants (empty circle) and 75 male infants (empty square) within the pre-specified PMA windows. Compared to male infants, regardless of gestational age at delivery, female infants had significantly higher leptin levels at a PMA of $26-29$ weeks ( $\left.{ }^{*} P<0.01\right), 30-32$ weeks $\left({ }^{*} P<0.05\right)$, and $33-36$ weeks $\left({ }^{* *} P<0.01\right)$

17 due to early discharge before 36 weeks PMA. Also, no blood sample was available from 14 infants at 36 weeks PMA, leaving 104 infants. Median leptin level at 36 weeks PMA was $1108 \mathrm{pg} / \mathrm{ml}$. Thirty-six week PMA leptin level predictor variables and their median or proportion are represented in Table 3. GA at birth, sex, age, weight, weight Z-score, and BPD were all independent predictors of infant leptin levels at 36 weeks PMA (Table 3). Multivariate analysis was performed with those significant variables, except age in days and weight Z-score, as those strongly correlate with GA at birth and weight at 36 weeks PMA, respectively. Infant's sex and weight remained significant predictors of infant plasma leptin levels at 36 weeks PMA (Table 3), with weight having positive correlation with leptin levels and female infants having higher leptin levels than male infants (1213 $\mathrm{pg} / \mathrm{ml}$ vs. $984 \mathrm{pg} / \mathrm{ml}$ ). Caloric intake from breast milk and the form of nutrition did not predict leptin levels, although a vast majority of infants received predominately breast milk by the time they reached 36 weeks gestation.

\section{DISCUSSION}

In this study, we describe postnatal leptin levels in preterm infants from periviable birth until near term gestation. We utilized cord blood leptin levels as a surrogate for the levels typically seen in the developing fetus. To our knowledge, this is the most comprehensive study describing postnatal leptin level changes in a preterm population with comparison to "reference fetus" levels. Prior studies have shown that preterm infants have lower leptin levels than term infants. ${ }^{10,11}$ This is not surprising, as there are many studies, including ours, demonstrating that cord blood leptin levels increase with advancing gestation. $6,7,15$ Therefore, it would be unfair to expect that preterm infants at birth have similar leptin levels to those of term infants. However, the fact that leptin levels in preterm infants remain low for weeks, ${ }^{11,16}$ and this low leptin state can be observed even past term gestation, ${ }^{10,17}$ confirms that preterm infants have profound and persistent leptin deficiency. To our knowledge, Hellgren and coworkers were the first to highlight this concern, by comparing preterm infants postnatal leptin levels with other preterm infants, instead of full term infant levels. ${ }^{18}$ In their study, infants born at 28 weeks were found to have lower leptin at 32 weeks PMA than infants born at 32 weeks. Our study provides deeper insight into preterm infant postnatal leptin level changes, and it is the first to describe the depth and duration of leptin deficiency in highly vulnerable periviable infants.

The most commonly reported cord blood leptin modulators are GA and fetal weight, specifically fetal adiposity. ${ }^{15,19}$ Despite fetal adipocytes producing leptin as early as 20 weeks gestation, ${ }^{20}$ adipocytes are less responsive to other hormonal influences and are not present in all major fat deposition areas until around 28 weeks gestation. ${ }^{21}$ Thus cord blood leptin levels increase slowly from 18 to 32 weeks gestation, but accumulation of fetal mass after 32 weeks $^{22}$ drives a late gestation leptin surge. 6,7 There are studies that did not identify a relationship between GA and cord blood leptin levels in preterm populations, but statistical power was limited by the sample size. ${ }^{15,23}$ Despite evaluating leptin levels in infants born before significant adipose mass accumulation, we show positive correlation between GA and leptin levels. Presence of this association in earlier gestations is understandable from a developmental standpoint, as there are redundant systems in place to ensure the adequacy of circulating fetal leptin levels, including a critical role for increasing maternal weight gain and leptin production during pregnancy.

As fetal immaturity limits autologous leptin synthesis in the first two trimesters of pregnancy, it is not unexpected that cord blood leptin levels are affected by maternal factors. Although we did not measure maternal leptin or body composition directly, we did assess multiple maternal clinical variables as predictors for cord blood leptin. In our study, we evaluated maternal first trimester $\mathrm{BMI}, \mathrm{BMI}$ at delivery, and maternal weight gain during pregnancy. The highest weight gain was noted in the group of mothers that delivered at 30-32 weeks gestation. The most likely explanation is that these mothers were further along in the pregnancy; thus they had more time for pregnancy weight gain. Even after adjusting for GA at time of birth, maternal BMl at time of delivery and maternal pregnancy weight gain were both independent predictors of cord blood leptin. There are studies showing similar findings ${ }^{23,24}$ and maternal-fetal leptin transfer has been previously demonstrated. Notably, our cohort's median BMI $\left(26.2 \mathrm{~kg} / \mathrm{m}^{2}\right)$ is lower than the average BMI of woman in America between the ages 20 and 39 years $\left(28.7 \mathrm{~kg} / \mathrm{m}^{2}\right)$, both values are considered overweight. ${ }^{25}$ Maternal influence on fetal leptin levels is supported by association between maternal comorbidities, like diabetes, on cord blood leptin levels. ${ }^{7,17}$

It appears that maternal factors also may have a direct effect on placental leptin secretion. For example, increased placental leptin mRNA and protein expression has been described in mothers with diabetes, preeclampsia, and chorioamnionitis. ${ }^{26-28}$ Knowing that there is a direct placental leptin secretion, ${ }^{20}$ we evaluated the effect of maternal morbidity on cord blood leptin levels, but did not observe statistically significant effects. Of note, we did remove outliers from the variate analysis, and the mother of the 30-32 week infant with a cord blood level of $17,459 \mathrm{pg} / \mathrm{ml}$ carried a diagnosis of Diabetes Mellitus and was morbidly obese, with a first trimester BMI of 41 . After birth, the affected infant's leptin levels remained extremely high, but did decrease in a similar fashion as other infants $(17,459 \mathrm{pg} / \mathrm{ml}$ to $8,041 \mathrm{pg} / \mathrm{ml}$ within $24 \mathrm{~h}$, to $2,567 \mathrm{pg} / \mathrm{ml}$ within $48 \mathrm{~h}$, and to $538 \mathrm{pg} / \mathrm{ml}$ within $72 \mathrm{~h}$ ). Further preclinical and clinical studies would be beneficial to evaluate how antenatal complications, and perhaps genetic or environmentally induced leptin resistance, affect fetal leptin levels.

Trans-placental leptin delivery is reportedly influenced by steroid exposure. Preclinical investigations in rats have shown 
Table 3. Plasma leptin levels at 36 weeks postmenstrual age $(P M A)(N=104)$ were correlated with maternal and infant variables by univariate linear regression followed by generalized linear modeling to determine independent predictors

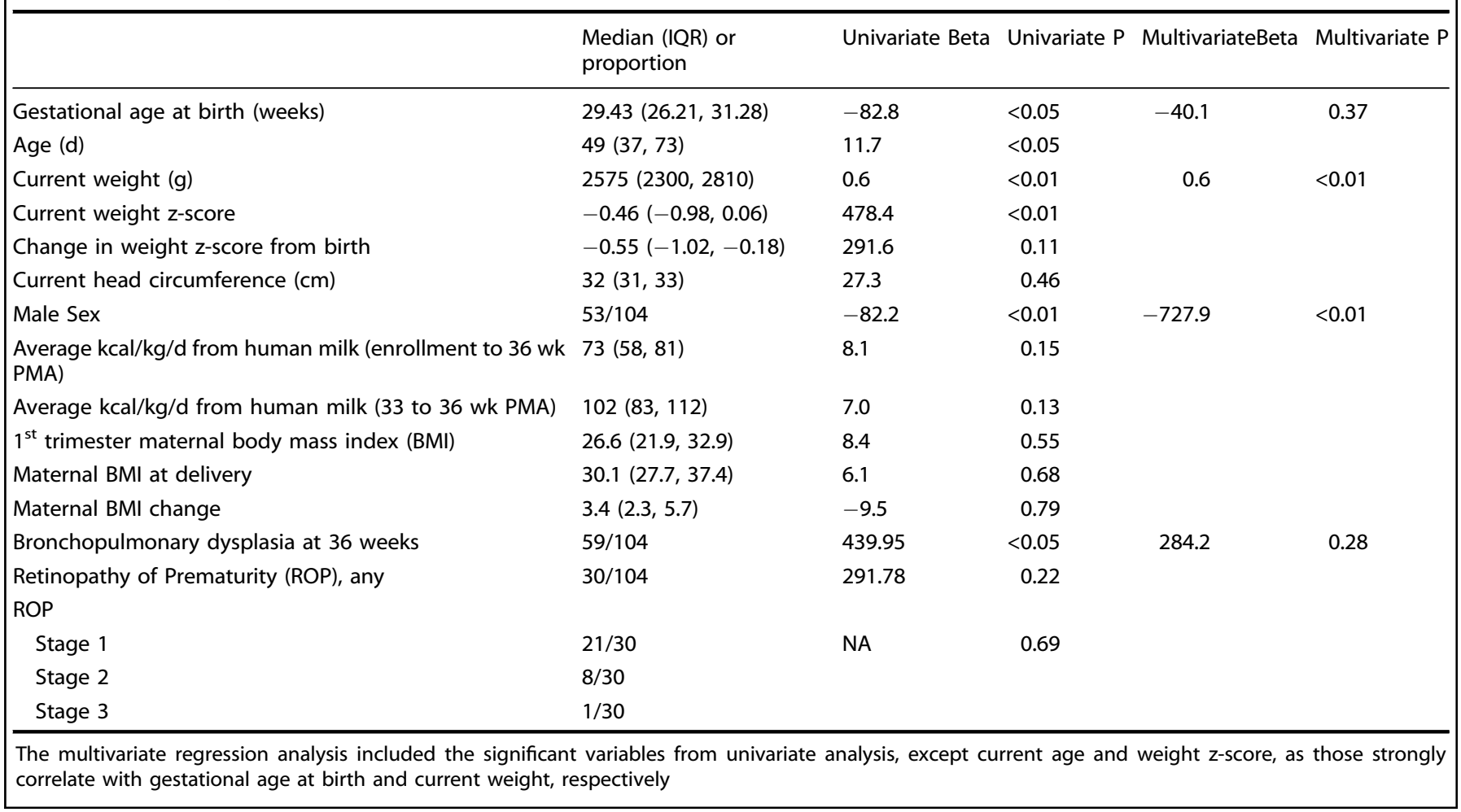

decreased trans-placental leptin delivery after antenatal dexamethasone. ${ }^{29}$ Subsequently, similar findings with antenatal betamethasone administration were shown in clinical studies. ${ }^{30}$ Unfortunately, we were not able to further evaluate antenatal steroid effects, as $96 \%$ of our study population were exposed to steroids. Although exploratory analysis showed a trend towards lower leptin levels after an incomplete course of steroids, this did not reach statistical significance, and it is unclear if the association could be related to an acute suppressive effect of the initial steroid dose on placental leptin transfer and/or a stimulatory effect of $>24 \mathrm{~h}$ steroid exposure on adipocyte leptin production. The latter possibility is supported by the increased leptin levels seen during postnatal dexamethasone exposure, but our study was not designed to test those hypotheses and our results did not reach the threshold for statistical significance. Besides antenatal steroids, other adrenal and gonadal steroids affect leptin levels, with male fetuses having lower leptin levels than females. ${ }^{9,31}$ There is no clear evidence explaining this sex dimorphism, but there is a negative relationship between testosterone and leptin in adult male populations, ${ }^{32}$ and a similar association has been described in preterm infants. ${ }^{11}$ Furthermore, testosterone directly decreases leptin mRNA expression in adipocytes. ${ }^{33}$ In contrast, estrogen has been shown to directly increase leptin mRNA expression, ${ }^{34}$ and a positive association between estrogen and leptin has been described in some but not all clinical studies. ${ }^{35,36}$ In our study, cord blood leptin levels were not affected by infant's sex, but there was a marked difference between postnatal leptin levels in males and females from 26 to 36 weeks PMA.

Following delivery, preterm infant leptin levels fall abruptly. Postnatal decreases in leptin are also seen in term infants, albeit to a lesser extent. ${ }^{24,37}$ This initial drop may represent acute placental and maternal supply loss. Infants born at 22 to 29 weeks gestation exhibit the steepest drop, suggesting higher dependence on trans-placental leptin. Furthermore, their leptin levels remain low for weeks, and there is no significant rise until after 32 weeks PMA, signifying immaturity of adipose tissue. Interestingly, after 32 weeks PMA leptin levels increase more distinctly in these infants. Possibly, this represents altered body composition with increased fat mass accumulation, as is frequently seen in preterm infants. ${ }^{38}$ Overall, postnatal leptin changes in the preterm population parallel the leptin level changes seen in fetuses using cord blood, but it appears that their third trimester leptin surge occurs a few weeks later than that seen from cord blood, where the increase is seen already starting 30 weeks PMA. It is also noteworthy that cordocentesis studies have confirmed findings in animal models that demonstrate increasing intrauterine leptin levels throughout gestation. ${ }^{7,39}$ Further supporting the contention that cord blood levels reflect intrauterine values rather than delivery-induced aberrations, intrauterine cordocentesis levels are very consistent with cord blood levels obtained immediately after delivery at the corresponding PMA., 15,40 Some could argue that the low leptin levels are a protective mechanism for survival in infancy, to enhance hunger and expansion of energy stores needed for rapid growth. Thus, this low leptin state in preterm population might be part of normal postnatal adaptation, since term infant leptin levels are below cord levels even at 2 years of age. $^{37}$ In contrast, preterm infants significantly differ from those born at term, as organ maturation has not yet occurred, and they must undergo major developmental changes in the extrauterine environment.

Although univariate analysis suggested that the diagnosis of BPD predicted higher leptin levels at 36 weeks PMA, that relationship was not seen on multivariate analysis. Male sex and current weight were the primary predictors of 36 week leptin levels, and it is possible sex and weight confounded the association between leptin and BPD. Alternatively, it is possible that leptin has effects on surfactant production, alveolar development and fibrosis, but the effect of leptin on the human lung appear to be less beneficial than the positive effects often seen in preclinical models. ${ }^{41,42}$ 
Maternal breast milk intake has been associated with improved intelligence, suggesting the presence of protective factors in breast milk. Leptin has been identified in maternal breast milk ${ }^{43}$ and the role of breast milk delivery of leptin in the maintenance of term infant leptin levels is supported by studies showing that breastfed infants have higher leptin levels than formula-fed babies. ${ }^{12}$ Animal studies have shown that leptin is absorbed enterally. ${ }^{44}$ To our knowledge, there are no studies evaluating the breast milk effect on preterm infant leptin levels. Unfortunately, we were not able to assess if infant's leptin levels are affected by feeding type as all infants received breast milk at some point during their NICU stay. Similarly, we were not able to evaluate if leptin levels are influenced by the type of breast milk an infant receives (maternal vs donor), as there were no infants receiving exclusively donor breast milk. We did evaluate if the amount of maternal breast milk infants received affects their leptin levels at 36 weeks PMA, and did not observe any association, but our study is limited in that we did not measure the leptin concentration in the breast milk. The average caloric intake from human milk reported in Table 3 underestimates total caloric intake because it includes breast milk intake via feeding tube or bottle, but excludes breastfeeding due to inability to accurately quantify breastfeeding volumes, which is a limitation of this study. All breast milk is not equal, as leptin content in maternal breast milk varies. ${ }^{43}$ Therefore, based on our results, we cannot disregard maternal breast milk as a potential route of leptin supplementation. That said, addition of leptin to parenteral nutrition would be the most reliable method of leptin delivery in the postnatal period, but as is the case for infant formulas, those solutions currently do not contain leptin. ${ }^{45}$

Our study has several additional limitations. We did not obtain maternal blood samples or longitudinal samples from all infants, as research samples were collected only with routine lab draws. We do not have information on the infants' body composition, and they were not randomized to receive maternal breast milk vs donor breast milk vs another form of nutrition.

In conclusion, preterm delivery is associated with rapid onset and profound leptin deficiency that is sustained through 36 weeks PMA. The pattern of leptin level decline and recovery is influenced by sex and GA at birth. This prolonged low leptin state might represent functional immaturity, lack of sufficient adipose tissue or suboptimal nutrition. Future studies are needed to evaluate if this low leptin state skews the developmental processes during critical windows of susceptibility, resulting in neurodevelopmental implications. Targeted nutritional interventions may be needed to improve the postnatal leptin status and development of premature infants.

\section{ACKNOWLEDGEMENTS}

Authors would like to thank Claire Goeke and Jacky Walker for their tremendous help with sample acquisition, and Elizabeth Kenkel for her support with Multiplex analysis. This work would not be possible without the enthusiastic support we received from NICU families, bedside nurses, and the NICU phlebotomy team. This study was supported by the Children's Miracle Network and the National Center for Advancing Translational Sciences of the National Institutes of Health (ULITR002537).

\section{AUTHOR CONTRIBUTIONS}

B.S.: Conception and design, acquisition of data, and analysis and interpretation of data; drafting and revising the article for important intellectual content; and final approval of the version to be published. T.T.C.: Conception and design and analysis and interpretation of data; revising the article for important intellectual content; and final approval of the version to be published. L.V.: Acquisition of data; revising the article for important intellectual content; and final approval of the version to be published. K.J.J.: Conception and design and acquisition of data and final approval of the version to be published. DAS: Acquisition of data and final approval of the version to be published. S.E.H.: Interpretation of data; revising the article for important intellectual content; and final approval of the version to be published. R.D.R.:
Conception and design and analysis and interpretation of data; revising the article for important intellectual content; and final approval of the version to be published

\section{ADDITIONAL INFORMATION}

Competing interest: The authors declare no competing interest.

Publisher's note: Springer Nature remains neutral with regard to jurisdictional claims in published maps and institutional affiliations.

\section{REFERENCES}

1. Nasri, H. Z., Houde, Ng. K., Westgate, M. N., Hunt, A. T. \& Holmes, L. B. Malformations among infants of mothers with insulin-dependent diabetes: is there a recognizable pattern of abnormalities? Birth Defects Res. 110, 108-113 (2018).

2. Meyer, L. R., Zhu, V., Miller, A. \& Roghair, R. D. Growth restriction, leptin, and the programming of adult behavior in mice. Behav. Brain Res. 275, 131-135 (2014).

3. Meier, U. \& Gressner, A. M. Endocrine regulation of energy metabolism: review of pathobiochemical and clinical chemical aspects of leptin, ghrelin, adiponectin, and resistin. Clin. Chem. 50, 1511-1525 (2004).

4. Ahima, R. S., Bjorbaek, C., Osei, S. \& Flier, J. S. Regulation of neuronal and glial proteins by leptin: implications for brain development. Endocrinology 140, 2755-2762 (1999).

5. Steppan, C. M. \& Swick, A. G. A role for leptin in brain development. Biochem Biophys. Res Commun. 256, 600-602 (1999).

6. Stoll-Becker, $\mathrm{S}$. et al. Influence of gestational age and intrauterine growth on leptin concentrations in venous cord blood of human newborns. Klin. Padiatr. 215, 3-8 (2003).

7. Cetin, I. et al. Fetal plasma leptin concentrations: relationship with different intrauterine growth patterns from 19 weeks to term. Pediatr. Res. 48, 646-651 (2000).

8. Steinbrekera, B., . \& Roghair, R. Modeling the impact of growth and leptin deficits on the neuronal regulation of blood pressure.J. Endocrinol. 231, R47-R60 (2016).

9. Helland, I. B., Reseland, J. E., Saugstad, O. D. \& Drevon, C. A. Leptin levels in pregnant women and newborn infants: gender differences and reduction during the neonatal period. Pediatrics 101, E12 (1998).

10. Toprak, D. et al. Serum leptin levels of premature and full-term newborns in early infancy: metabolic catch-up of premature babies. Turk. J. Pediatr. 46, 232-238 (2004).

11. Ertl, T. et al. Postnatal changes of leptin levels in full-term and preterm neonates: their relation to intrauterine growth, gender and testosterone. Biol. Neonate 75, 167-176 (1999).

12. Savino, F. et al. Looking for a relation between serum leptin concentration and body composition parameters in healthy term infants in the first 6 months of life. J. Pediatr. Gastroenterol. Nutr. 46, 348-351 (2008).

13. Santillan, M. K. et al. "Collection of a lifetime: a practical approach to developing a longitudinal collection of women's healthcare biological samples". Eur. J. Obstet. Gynecol. Reprod. Biol. 179, 94-99 (2014).

14. Shennan, A. T., Dunn, M. S., Ohlsson, A., Lennox, K. \& Hoskins, E. M. Abnormal pulmonary outcomes in premature infants: prediction from oxygen requirement in the neonatal period. Pediatrics 82, 527-532 (1988).

15. Jaquet, D., Leger, J., Levy-Marchal, C., Oury, J. F. \& Czernichow, P. Ontogeny of leptin in human fetuses and newborns: effect of intrauterine growth retardation on serum leptin concentrations. J. Clin. Endocrinol. Metab. 83, 1243-1246 (1998).

16. Ohkawa, N. et al. IGF-I, leptin and active ghrelin levels in very low birth weight infants during the first 8 weeks of life. Acta Paediatr. 99, 37-41 (2010).

17. Spear, M. L. et al. Immaturity or starvation? Longitudinal study of leptin levels in premature infants. Biol. Neonate 80, 35-40 (2001).

18. Hellgren, G., Engstrom, E., Smith, L. E., Lofqvist, C. \& Hellstrom, A. Effect of preterm birth on postnatal apolipoprotein and adipocytokine profiles. Neonatology 108, 16-22 (2015).

19. Clapp, J. F. 3rd \& Kiess, W. Cord blood leptin reflects fetal fat mass. J. Soc. Gynecol. Investig. 5, 300-303 (1998).

20. Lepercq, J. et al. Prenatal leptin production: evidence that fetal adipose tissue produces leptin. J. Clin. Endocrinol. Metab. 86, 2409-2413 (2001).

21. Poissonnet, C. M., LaVelle, M. \& Burdi, A. R. Growth and development of adipose tissue. J. Pediatr. 113, 1-9 (1988).

22. Lapillonne, A. et al. Body composition in appropriate and in small for gestational age infants. Acta Paediatr. 86, 196-200 (1997).

23. Ho, S. P. et al. Association of plasma leptin levels with maternal body weight and body mass index in premature and term newborns. Pediatr. Neonatol. 51, 19-25 (2010).

24. Valuniene, M. et al. Leptin levels at birth and in early postnatal life in small- and appropriate-for-gestational-age infants. Med. (Kaunas.) 43, 784-791 (2007). 
25. Fryar, C. D., Kruszon-Moran, D., Gu, Q. \& Ogden, C. L. Mean body weight, height, waist circumference, and body mass index among adults: United States, 1999-2000 through 2015-2016. National Health Statistics Reports; no 122. (National Center for Health Statistics, Hyattsville, MD, 2018).

26. Lea, R. G. et al. Placental leptin in normal, diabetic and fetal growth-retarded pregnancies. Mol. Hum. Reprod. 6, 763-769 (2000).

27. Mise, H. et al. Augmented placental production of leptin in preeclampsia: possible involvement of placental hypoxia. J. Clin. Endocrinol. Metab. 83, 3225-3229 (1998)

28. Taki, A. et al. Expression of angiogenesis-related factors and inflammatory cytokines in placenta and umbilical vessels in pregnancies with preeclampsia and chorioamnionitis/funisitis. Congenit. Anom. (Kyoto) 52, 97-103 (2012).

29. Smith, J. T. \& Waddell, B. J. Leptin distribution and metabolism in the pregnant rat: transplacental leptin passage increases in late gestation but is reduced by excess glucocorticoids. Endocrinology 144, 3024-3030 (2003).

30. Marinoni, E. et al. Effects of prenatal betamethasone administration on leptin and adiponectin concentrations in maternal and fetal circulation. Am. J. Obstet. Gynecol. 199, e141-e146 (2008).

31. Ong, K. K. et al. Cord blood leptin is associated with size at birth and predicts infancy weight gain in humans. ALSPAC Study Team. Avon Longitudinal Study of Pregnancy and Childhood. J. Clin. Endocrinol. Metab. 84, 1145-1148 (1999).

32. Behre, H. M., Simoni, M. \& Nieschlag, E. Strong association between serum levels of leptin and testosterone in men. Clin. Endocrinol. (Oxf.) 47, 237-240 (1997).

33. Wabitsch, M. et al. Contribution of androgens to the gender difference in leptin production in obese children and adolescents. J. Clin. Investig. 100, 808-813 (1997).

34. Machinal, F., Dieudonne, M. N., Leneveu, M. C., Pecquery, R. \& Giudicelli, Y. In vivo and in vitro ob gene expression and leptin secretion in rat adipocytes: evidence for a regional specific regulation by sex steroid hormones. Endocrinology 140, 1567-1574 (1999).
35. Bednarek-Tupikowska, G. et al. Serum leptin concentrations in pre- and postmenopausal women on sex hormone therapy. Gynecol. Endocrinol. 22, 207-212 (2006).

36. Hong, S. C. et al. Correlation between estrogens and serum adipocytokines in premenopausal and postmenopausal women. Menopause 14, 835-840 (2007).

37. Jaquet, D., Leger, J., Tabone, M. D., Czernichow, P. \& Levy-Marchal, C. High serum leptin concentrations during catch-up growth of children born with intrauterine growth retardation. J. Clin. Endocrinol. Metab. 84, 1949-1953 (1999).

38. Johnson, M. J., Wootton, S. A., Leaf, A. A. \& Jackson, A. A. Preterm birth and body composition at term equivalent age: a systematic review and meta-analysis. Pediatrics 130, e640-e649 (2012).

39. Nüsken, E. et al. Reduced perinatal leptin availability may contribute to adverse metabolic programming in a rat model of uteroplacental insufficiency. Endocrinology 157, 1813-1825 (2016).

40. Geary, M., Herschkovitz, R., Pringle, P. J., Rodeck, C. H. \& Hindmarsh, P. C. Ontogeny of serum leptin concentrations in the human. Clin. Endocrinol. (Oxf.) $\mathbf{5 1}$ 189-192 (1999).

41. King, G., Smith, M. E., Cake, M. H. \& Nielsen, H. C. What is the identity of fibroblastpneumocyte factor? Pediatr. Res. 80, 768-776 (2016).

42. Gui, X., Chen, H., Cai, H., Sun, L. \& Gu, L. Leptin promotes pulmonary fibrosis development by inhibiting autophagy via PI3K/Akt/mTOR pathway. Biochem. Biophys. Res. Commun. 498, 660-666 (2018).

43. Andreas, N. J. et al. Effect of maternal body mass index on hormones in breast milk: a systematic review. PLOS ONE 9, e115043 (2014).

44. Casabiell, X. et al. Presence of leptin in colostrum and/or breast milk from lactating mothers: a potential role in the regulation of neonatal food intake. J. Clin. Endocrinol. Metab. 82, 4270-4273 (1997).

45. Resto, M. et al. Leptin levels in preterm human breast milk and infant formula. Pediatrics 108, E15 (2001). 